angedeutet): Alsbaldige Verlegung in den Offenen Vollzug, damit die Arbeit wieder aufgenommen und die partnerschaftliche Beziehung gefestigt werden.

In der Interventionsprognose sind „passende“ Behandlungsvorschläge zu machen und solche auszuschließen, die nutzlos oder gar kontraproduktiv sind. So braucht nicht jeder, der ein Delikt im Alkoholrausch begangen hat, eine Entwöhnungsbehandlung (manchmal sind Rauschtaten geradezu Ausdruck einer Enthemmung wegen geringer Alkoholtoleranz und somit Beleg einer nicht vorhandenen Gewöhnung). Und nicht jeder Gewalttäter ist im Anti-Agressionstraining richtig. Solche, die sich am Leiden anderer ergötzen, hätten dort nur einen Lustgewinn.

Bisher hat die MIVEA bei meiner Arbeit mit Wiederholungstätern immer „gepasst“ und mir (und damit den Probanden) zu Erkenntnissen verholfen auf die ich ohne die Methode nicht gekommen wäre. Ich will aber nicht unterschlagen, daß ich bei Vielfach-Inhaftierten in der Interventionsprognose manchmal „passen“ muss. Wenn einer seit seiner Kindheit rundum zum K-idealtypischen Verhalten neigt und eine deliktische Handlung an die andere reiht und deshalb schon mehrfach das volle Repertoire der strafrechtlichen und sozialpädagogischen Interventionen kennen lernen „durfte“, ohne daß sich etwas änderte, dann fällt mir auch mit der MIVEA nichts mehr ein.

\section{Literatur:}

GÖPPINGER, Hans (1985), Angewandte Kriminologie, Ein Leitfaden für die Praxis, Berlin 1985

GÖPPINGER, Hans (2008), Kriminologie, 6. Auflage, München 2008

BOCK, Michael (1984), Kriminologie als Wirklichkeitswissenschaft, Berlin 1984

BOCK, Michael (2007), Kriminologie, 3. Auflage, München 2007

WEBER, Max (1988), Gesammelte Aufsätze zur Wissenschaftslehre, Tübingen 1988.

Jürgen Oetting ist Soziologe und Sozialtherapeut und arbeitet in der JVA Kiel.

\title{
Jugendtypisches Fehlverhalten im Straßenverkehr
}

Denise Kühn

Nicht zuletzt durch das zum 01.08.2007 in Kraft getretene Gesetz zur Einführung eines Alkoholverbots für Fahranfänger und Fahranfängerinnen ist insbesondere die Rolle der Heranwachsenden im Straßenverkehr in das Blickfeld des öffentlichen Interesses gerückt. Hauptziel des $\$ 24 \mathrm{c}$ StVG ist die Verringerung des besonders für junge Fahrer bestehenden hohen Unfallrisikos in der Probezeit. Es kommt nicht selten vor, dass Jugendliche oder Heranwachsende mit dem Erwerb der Fahrerlaubnis meinen, auch tatsächlich die uneingeschränkte Fähigkeit zum sicheren Führen eines Fahrzeugs erreicht zu haben. Kommt es dennoch zu Fehlverhalten im Straßenverkehr und anschließenden Verfahren, schließen sich manche Vertreter der formellen Sozialkontrolle ebenfalls dieser Auffassung an. Doch was sind die Ursachen für aggressive Verhaltensweisen und wie gehen speziell junge Fahranfänger hiermit um? Im Folgenden soll die Situation junger Fahranfänger verdeutlicht werden, deren Schwierigkeiten in dieser Anfangsphase oftmals die Anwendung von Jugendstrafrecht auf Heranwachsende rechtfertigt.

\section{Aggressive Fahrweise und situative Umstände}

\section{Entstehungsbedingungen von Aggressionen im Allgemeinen}

Aggression kann als ,jedes direkte oder indirekte Verhalten bezeichnet werden, durch das jemand einem anderen körperlich, geistig-seelisch oder auch gegenüber seinem Eigentum zu schaden droht, zu schaden versucht oder in der Tat mit Absicht schadet; Aggressivität wäre dann die in einem Menschen relativ dauerhaft vorhandene Bereitschaft in dieser Richtung. " 1 Der ethologische Ansatz zur Erklärung von Aggressionen basiert auf der Annahme, dass jeder Mensch mit einem angeborenen Aggressionstrieb auf die Welt kommt, berücksichtigt aber nicht, dass aggressives Verhalten häufig erst durch soziale Kontakte erlernt wird. Auch die psychoanalytische Theorie geht von einem angeborenen Aggressionstrieb aus, kann aber aufgrund der Vernachlässigung sozialer Faktoren ebenfalls keine umfassende Erklärung für die Entstehung aggressiver Verhaltensweisen liefern. Aus der Psychologie stammt die Erkenntnis, dass ein enger Zusammenhang zwischen Frustration und Aggression besteht. Die Frustrations-Aggressions-Theorie von John Dollard stellt daher darauf ab, dass jede Form von Frustration in Form erlebter Zurückweisungen zur Aggression (behavioristische Lerntheorie) führt. Michael Gottfredson und Travis Hirschi führen wiederum eine mangelnde Selbstkontrolle („low-self-control“) als Ursache aggressiver Entladungen an. Nach der sozial-kognitiven Lerntheorie von Albert Bandura hingegen sollen aggressive Verhaltensweisen durch Beobachtung des Verhaltens anderer, insbesondere von Bezugspersonen wie Eltern und Autoritäten in sog. „peer-groups“ oder Medienfiguren, und der sich hieraus für das Individuum ergebenden Konsequenzen (Lernen am Modell) erlernt werden. ${ }^{2}$ Das Beobachtungslernen beeinflusst nicht nur kurzfristig die Häufigkeit aggressiven Verhaltens, sondern führt zu einer mittel- und langfristigen Verhaltensprägung; das lerntheoretische Modell hebt dabei besonders gut die kognitiven Aspekte des Lernens hervor und versteht den menschlichen Lernvorgang als einen „aktiven, kognitiv gesteuerten seelischen Verarbeitungsprozess, bei dem komplexe Denkprozesse eine entscheidende Rolle spielen".3

\section{Aggressionen im Straßenverkehr}

Im Straßenverkehr äußert sich aggressives Verhalten sehr verschieden, genannt sei z.B. intensives Beschleunigen und Nötigungen in Form von Drängeleien ${ }^{4}$, Überholmanöver in der Kolonne, Dazwischendrängeln, Beschleunigen beim Überholtwerden, abrupter Spurwechsel, Schneiden eines anderen Fahrzeugs usw. Dabei sind das 
Ausmaß und die Art der Erscheinung verschiedenartig, je nachdem, ob man den Straßenverkehr innerorts, auf Land- oder Autobahnstraßen beleuchtet. Bei den unterschiedlichen Erscheinungsformen und Entstehungsbedingungen von Aggressionen im Straßenverkehr sind die gleichen Zusammenhänge anzutreffen, die auch für Aggressionen im Allgemeinen kennzeichnend sind. Charakteristisch ist zunächst, dass es die jeweils andere Gruppe von Verkehrsteilnehmern ist, die aus der Sicht der einen Gruppe als besonders aggressiv betrachtet wird. ${ }^{5}$ So ärgert sich z.B. der schneller Fahrende über den Langsameren und derjenige, der lieber langsamer fährt über den Raser. Als Erklärungsansatz für aggressives Verhalten im Straßenverkehr wird z.B. das allgemeine Erregungsniveau hervorgehoben, dass z.B. selbst derjenige, der es wirklich nicht eilig hat, sobald er sich aber im Straßenverkehr bewegt, in eine allgemeine Eile gerät; der Straßenverkehr daher als immense Stresssituation mit Zeitdruck, Reizüberflutung und Hektik empfunden wird. ${ }^{6}$ Weitere zu nennende Aspekte sind z.B. die technisch bedingte Distanz und Anonymität im Straßenverkehr mit der Folge einer zwischenmenschlichen Entfremdung, die ständige Frustration im heutigen Massenverkehr, den $\mathrm{Zu}$ sammenhang von Fahrstil und bestehenden technischen Möglichkeiten, die Emotionalität der Fahrer in Bezug auf ihr Kraftfahrzeug und ihre Leistungsfähigkeit, den Zusammenhang mit allgemeinen Konkurrenz- und Durchsetzungstendenzen im gesellschaftlichen Leben sowie eine Unmenge an situativen Bedingungen, wie z.B. schlechte Witterungsverhältnisse oder anstrengende Beifahrer. ${ }^{7}$ Diese Einflussfaktoren führen schließlich dazu, dass zwischen einem und zwei Drittel aller Verkehrsunfälle durch aggressive Verhaltensweisen verursacht werden. ${ }^{8}$

\section{Die Position junger Täter im Unfallgeschehen}

\section{Warum haben junge Fahrer ein besonders hohes Unfallrisiko?}

Junge Fahranfänger sind seit Jahren die hauptsächliche Unfallgruppe im Straßenverkehr. Sie haben ein mehrfach höheres Unfallrisiko als erfahrene Fahrer ab 25 Jahre. Junge Fahrer bilden keine einheitliche Gruppe. Manche fahren vorsichtig und vermeiden Risiken. Andere suchen Spaß und Abenteuer. Sie praktizieren gefährliche Verhaltensweisen, mit denen sie z.B. imponieren wollen. Trotz aller Unterschiede haben sie gemeinsame Merkmale- ihre mangelnde Fahrerfahrung und ihre Jugendlichkeit. Fahranfänger haben noch längere Zeit nach der Ausbildung Schwierigkeiten, das Fahrzeug in kritischen Situationen zu beherrschen. Manche vertrauen auch zu stark auf die Sicherheit ihrer Fahrzeuge, fahren zu risikoreich und sorglos. Wahrnehmungsstrategien und Automatismen in Bezug auf die Fahrzeugbeherrschung sind noch nicht ausgeprägt. ${ }^{9}$ Dadurch sind sie sowohl als Verursacher als auch als Geschädigte gleichsam gefährdet.

In der Jugendphase stehen junge Menschen überdies vor besonderen Schwierigkeiten. Genannt seien beispielsweise Begriffe die Identitätssuche (als gleichwertiges erwachsenes Mitglied in der Gesellschaft anerkannt zu werden bzw. Beachtung zu finden), Ablösung vom Elternhaus bzw. das Streben nach Autonomie, Abenteuer und Statussymbolen, Problemflucht, Aufbegehren und Protest, eine labile Gefühlswelt und der nicht zu vernachlässigende Gruppeneinfluss. ${ }^{10}$ Dies wirkt sich nicht zuletzt auch auf das Verhalten im Straßenverkehr aus. Repräsentative Studien unter 10000 Teilnehmern belegten, dass gerade die Altersgruppe der 20-24jährigen die höchste Ausprägung aggressiver Verhaltensweisen im Straßenverkehr aufweist, während sie mit zunehmendem Alter abnimmt. ${ }^{11}$

\section{Welche Situationen sind für junge Fahrer besonders gefährlich?}

Besonders riskant sind Situationen, in denen sich mangelnde Fahrerfahrung und Jugendlichkeit stark auf das Fahrverhalten auswirken. Beim Testen eines neuen, unbekannten Fahrzeugs und beim Erproben der eigenen Fahrfähigkeiten wächst die Unfallgefahr, wenn z.B. die Aufmerksamkeit zu sehr auf das Fahrzeug und weniger auf die Verkehrssituation gerichtet ist. In ihrer jugendlichen Unbekümmertheit erkennen viele nicht die Grenzen ihrer Leistungsfähigkeit, überschätzen ihr Können und sind dann besonders überfordert. Sie schätzen die Folgen ihres Handelns weniger langfristig und differenziert ab. ${ }^{12}$ Es fällt ihnen schwer, die richtige Handlungsalternative zu wählen, gerade in Situationen, in denen der Gruppendruck zu riskanten Fahrmanövern animiert. Daher spielt auch die Selbstkontrolle eine große Rolle, die durch die genannten Merkmale wesentlich vermindert wird. Gefährlich ist überdies die Einnahme von Alkohol oder Drogen. Wenn Alkohol getrunken wird, um Gefühle des Versagens und des Scheiterns zu überwinden, „sich einmal groß und mächtig zu erleben “, erhöht sich das Risiko aggressiver Entladungen. ${ }^{13}$ Der Gebrauch von Substanzen kann situationsspezifisch enthemmen, anregen, die vermeintliche Empfindung höherer Leistungsfähigkeit vermitteln, die Impulsivität steigern und zur Auslösung von Aggressionen beitragen, so dass es situationsbedingt leichter zur Begehung von Straftaten kommen kann, namentlich zu Aggressionsdelikten z.B. im Bereich der Verkehrsgefährdung, Unfallverursachung und unerlaubtem Entfernen vom Unfallort (Verkehrskriminalität). ${ }^{14}$

\section{Statistische Erkenntnisse}

Aggressives Fahrverhalten ist der Hauptfall der Straßenkriminalität männlicher Jugendlicher. Dagegen liegt der Anteil der Frauen an der Verkehrsdelinquenz knapp unter 10\%. ${ }^{15}$ Diese Diskrepanz könnte dadurch erklärt werden, dass weibliche Jugendliche Gewalt und Ärger eher gegen sich selbst richten, während männliche Jugendliche ihre Aggressionen eher "nach außen“ gegen andere ausdrücken. ${ }^{16}$ Bei Delikten, die im unmittelbaren Zusammenhang mit dem Straßenverkehr stehen, findet sich eine erhebliche Delinquenzbelastung der 14- bis unter 21-Jährigen insgesamt, wobei die Belastung Heranwachsender ungewöhnlich groß ist. ${ }^{17}$ Vorwiegend sind dies Delikte, die im Zusammenhang mit Unfällen wegen überhöhter Geschwindigkeit stehen, Trunkenheitsdelikte und Fahrens ohne Führerschein bzw. trotz entzogener Fahrerlaubnis. Bei Jugendlichen dominiert insbesondere das Fahren ohne Fahrerlaubnis ( $\$ 21$ StVG), während die Gruppe der Heranwachsenden vor allem wegen Alkoholverkehrsdelikten $\left(\mathbb{S} \int 316,315\right.$ c I Nr. 1a StGB) auffällt. ${ }^{18}$

Dies lässt sich nach Eisenberg auf die Tatsache zurückführen, dass die Höchstbelastung mit Straßenverkehrsunfällen jeweils am Beginn der Lern- und Fahrweise liegt, während mit zunehmender Fahrpraxis die relative Unfallhäufigkeit durchgängig geringer wird. ${ }^{19}$ Weiterhin ist zu berücksichtigen, dass die Belastung der jüngeren Anfänger höher als diejenige der altersmäßig älteren Anfänger ist. ${ }^{20}$ Merkmale aufgrund mangelnder Fahrerfahrung sind z.B. die fehlende Abschätzung von Risiken, Bestätigungsbedürfnis oder aber mangelndes Verantwortungsbewusstsein und insbesondere das Bemühen um Erreichen des Erwachsenenstatus. ${ }^{21}$ In der Altersgruppe der 18- bis 25- Jährigen fährt nach einer Continental-Studie z.B. jeder Zweite schneller als erlaubt; je älter der Fahrer, desto höher ist die Wahrscheinlichkeit, dass er sich an die Geschwindigkeitsbegrenzung hält. ${ }^{22}$ Folge hiervon ist, dass $33 \%$ der Unfälle mit Personenschaden, die auf nicht angepasste Geschwindigkeit zurückzuführen sind, 
auf das Konto junger Fahrer geht. ${ }^{23}$ Gerade zur Nachtzeit bzw. in den frühen Morgenstunden besteht für diese Altersgruppe eine erhöhte Unfallgefahr (sog. Diskounfälle). Ergänzend muss berücksichtigt werden, dass eine Vielzahl von Straßenverkehrsdelikten im Dunkelfeld liegen.

\section{Voraussetzungen der Anwendung von Jugendstrafrecht auf Heranwachsende als mögliche Folge verkehrskrimi- nellen Verhaltens}

Auf Heranwachsende wird Jugendstrafrecht angewendet entweder aufgrund ihres Entwicklungsstandes ( $\mathbb{1 0 5}$ I Nr.1 JGG) oder, weil es sich bei der Tat um eine Jugendverfehlung handelt $(\mathbb{S} 105$ I Nr.2 JGG). $\ 3$ JGG (\$12 OWiG) findet keine Anwendung, da Heranwachsende im Gegensatz zu den bedingt strafmündigen Jugendlichen vom Gesetzgeber als unbeschränkt strafmündig angesehen werden. \105 I Nr. 1 und Nr.2 JGG stehen zwar grundsätzlich gleichbedeutend nebeneinander; letzterer stellt jedoch eine Beweiserleichterung für den Richter insofern dar, als eine umfassende Erforschung der Persönlichkeit, wie sie für Nr. 1 notwendig ist, entbehrlich ist, soweit die Voraussetzungen der Nr. 2 gegeben sind. ${ }^{24}$

\section{1. \105 I Nr. 2 JGG}

\ 105 I Nr. 2 JGG erfordert zunächst eine Jugendverfehlung eines Heranwachsenden. Heranwachsender ist nach der Legaldefinition des $\mathbb{1} 1$ II JGG wer zur Zeit der Tat 18, aber noch nicht 21 Jahre alt ist. Nach der Rechtsprechung des BGH sind Jugendverfehlungen in erster Linie Taten, die schon „nach ihrem äußeren Erscheinungsbild die Merkmale jugendlicher Unreife aufweisen, jedoch können auch lediglich die Beweggründe der Tat und ihre Veranlassung diese als eine Jugendverfehlung kennzeichnen." 25 "Es komme darauf an, ob die konkret begangene Tat auf jugendlichen Leichtsinn, Unüberlegtheit oder soziale Unreife zurückzuführen ist. "26 Für jugendspezifisches Verhalten spricht auch eine mangelnde Fähigkeit an Ausgeglichenheit, Besonnenheit und Hemmungsvermögen. ${ }^{27}$ Die Beurteilung einer Straftat als Jugendverfehlung ist im wesentlichen Tatfrage, bei der dem Tatrichter ein weiter Beurteilungsspielraum zukommt. ${ }^{28}$ Weiterhin erforderlich ist eine umfassende Würdigung der äußeren Tatumstände sowie der Beweggründe des Täters. Auch Straßenverkehrsdelikte können unter $\ 105$ I Nr. 2 fallen..$^{29}$ Wesentlich ist, dass es hinsichtlich des äußeren Erscheinungsbilds und der Motivation gerade um Verhaltensweisen geht, wie sie bei Jugendlichen sehr oft vorkommen. Es mag zwar sein, dass das äußere Erscheinungsbild der jugendlichen Taten mit der Begehungsweise ähnlicher Delikte durch Erwachsene übereinstimmt. Doch sind „Jugendverfehlungen nicht nur Taten, die schon nach ihrem äußeren Erscheinungsbild die Merkmale jugendlicher Unreife aufweisen. “ ${ }^{30}$ Bei Heranwachsenden liegt es nahe, dass dieses Defizit auf noch nicht voller sozialer Reife beruht; Straftaten im Verkehr gehen vielfach aus affektgesteuertem, impulsiven Verhalten hervor. ${ }^{31}$ Zudem können Faktoren, wie eine positive Stimmungslage (Vorfreude) und äußere Reize (Musik) dazu führen, dass diese Fahrer sich selbstsicher fühlen und dadurch ihre Fahrfähigkeit überschätzen. Die Geschwindigkeit zu erhöhen, um sich spontan möglichen Problemen zu entziehen, ist beispielsweise eine typische Erscheinung einer stressbedingten Kurzschlusshandlung, was wiederum ein typisches Merkmal jugendlicher unsicherer Fahrweise in Stresssituationen darstellt. Verkehrsdelikte Heranwachsender, die aus „puberaler motorischer Enthemmung, aus Geltungsbestreben, Selbstbestätigungs- und Erlebnisdrang erwach- sen, können auch bei aller intellektueller Kenntnis der Verkehrsvorschriften typische Reifungsdelikte darstellen. " 32 Lediglich aufgrund der Tatsache, die Fahrerlaubnis zu besitzen, kann folglich nicht auf das Vorliegen von Erwachsenenreife geschlossen werden. Längerer Besitz der Fahrerlaubnis begründet die Anwendung des Erwachsenenrechts nie. ${ }^{33}$ Die in der Praxis oft vorzufindende Übung, bei Straßenverkehrsdelikten allgemeines Strafrecht anzuwenden, weil nur in diesem Fall eine Erledigungsmöglichkeit über einen Strafbefehl vorhanden ist, überzeugt wenig, da eine genaue Aufarbeitung der Hintergründe und Entstehungszusammenhänge auch in diesem Bereich auf jugendtypisches bzw. altersgemäßes Verhalten in einer Übergangszeit hinweist, so dass aus diesem Grunde Jugendstrafrecht zur Anwendung kommen sollte. ${ }^{34}$ Im Zweifel muss jedenfalls vom Jugendstrafrecht Gebrauch gemacht werden.

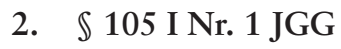

Voraussetzung ist gem. $\ 105$ I Nr. 1 JGG, dass die Gesamtwürdigung der Persönlichkeit des Täters bei Berücksichtigung auch der Umweltbedingungen ergibt, dass er zur Zeit der Tat nach seiner sittlichen und geistigen Entwicklung noch einem Jugendlichen gleichstand. Es kommt nach der Rechtsprechung maßgeblich darauf an, ob es sich bei dem Heranwachsenden um einen noch ungefestigten, in der Entwicklung stehenden und noch prägbaren Menschen handelt, bei dem Entwicklungskräfte noch in größerem Umfang wirksam sind. ${ }^{35}$ Dabei ist jedoch nicht ein Vergleich mit einem imaginären durchschnittlichen Heranwachsenden zu ziehen und auf zu beobachtende Reifeverzögerungen und Retardierungen zu achten. ${ }^{36}$ Entscheidend ist vielmehr die individuelle Reifeentwicklung des Heranwachsenden, wobei es vor allem um die Fähigkeit zu planendem, in die Zukunft gerichteten Denken und Handeln sowie um das Verhalten anderer Menschen gegenüber geht. Insoweit lässt sich in der Praxis eine Tendenz zur Annahme von Jugendstrafrecht konstatieren, ohne dass die Anwendung von Jugend- oder Erwachsenenstrafrecht rechtlich im Verhältnis von Regel und Ausnahme stünde. ${ }^{37}$ Die Formel der Rechtsprechung ist allerdings unbestimmt und ermöglicht keine eindeutige Zuordnung. Daher wird auf das Vorliegen bestimmter Indikatoren für die Reifeverzögerung zurückgegriffen, wie z.B. das frühzeitige Verlassen des Elternhauses sowie die Ablösung von bisherigen Bezugsgruppen und die Übernahme neuer Rollen in Partnerschaft und Beruf; Drogenabhängigkeit, welche regelmäßig die soziale Reifung verzögert, Gruppendelinquenz, sofern darin eine Anpassungs- bzw. Gehorsamsbereitschaft zum Ausdruck kommt oder Störungen der psychosozialen Entwicklung in der Kindheit und Jugend. ${ }^{38}$ Im Schrifttum wird vielfach noch auf die sog. Marburger Richtlinien zurückgegriffen, welche sich auf jugendtümliche Kriterien beziehen, wie z.B. planloses impulsives situationsbedingtes Handeln, Geltungsbedürfnis, Leichtsinn, Unbekümmertheit, naivvertrauensseliges Verhalten, eine spielerische Einstellung zur Arbeit oder Erlebnishunger. ${ }^{39}$

Auch bei Straßenverkehrsdelikten spielen entwicklungsbeeinflusste Merkmalsausprägungen wie fehlende Abschätzung von Risiken, Bestätigungsbedürfnis oder mangelndes Verantwortungsbewusstsein sowie das Bemühen um das Erreichen des Erwachsenenstatus eine Rolle. ${ }^{40}$ Allerdings deutet nicht selten das Verhalten Heranwachsender auf planlose, impulsive und situationsbedingte Handlungsweisen, Leichtsinn, Unbekümmertheit, Geltungsbedürfnis und Erlebnishunger hin und damit auf ein jugendtümliches Verhalten. 


\section{Abschließende Betrachtung}

$\mathrm{Zu}$ fragen bleibt nun, welche Sanktion in Folge der Begehung von Straßenverkehrsdelikten in Betracht kommt. Im jugendgerichtlichen Verfahren kann ein Fahrverbot gem. \$8 III i.V.m. \$\$2, 76 S. 1 JGG; 44 StGB nicht nur neben einer Jugendstrafe, sondern auch neben Erziehungsmaßregeln und Zuchtmitteln verhängt werden sowie neben der Aussetzung der Verhängung der Jugendstrafe gem. \ 27 JGG. Möglich erscheint aber auch die Anordnung der Entziehung der Fahrerlaubnis aus erzieherischen bzw. spezialpräventiven Gesichtspunkten gem. $\int \mathbb{S} 8$ III, 7 JGG; 69 StGB. Wie bereits festgestellt, kann die Aburteilung der Begehung von Straßenverkehrsdelikten bei Heranwachsenden in den Anwendungsbereich des $\$ 105$ JGG fallen, so dass die Verhängung eines Fahrverbots nach Erwachsenenstrafrecht zwar möglich, aber nicht zwingend ist. Gelangt aber allgemeines Strafrecht zur Anwendung, ist dies jedoch nicht als nützliche oder sinnvolle Sanktion anzusehen. Gerade hinsichtlich junger Delinquenten sollten Sanktionen eher unterstützend wirken als rein restriktiv, um eine positive Entwicklung der Jugendlichen bzw. Heranwachsenden zu verantwortungsbewussten Mitgliedern der Gesellschaft zu unterstützen. Im Falle der Verhängung eines Fahrverbots werden junge Fahranfänger am Verfestigen ihrer Fahrfähigkeiten gehindert und dadurch bei einem späteren Eintritt eine unsichere Fahrweise aufzeigen, so dass hierdurch keine Verbesserungen im Verkehrsverhalten zu erwarten sind. Daher sollten in erster Linie Weisungen i. S. d. \10 JGG angewendet werden, wie z.B. die Teilnahme an einem Verkehrsunterricht (Nr.9). Vorzuschlagen wäre i. R. d. offen formulierten $\$ 10$ JGG überdies, dem verkehrsdelinquenten Heranwachsenden aufzuerlegen, eine gewisse Zeit lang in Begleitung einer „verkehrserfahrenen“ Vorbildperson zu fahren, entsprechend dem begleiteten Fahren ab 17. Auf diese Weise bietet sich dem jungen Erwachsenen eine Möglichkeit, Erfahrung und Sicherheit hinsichtlich des eigenen Fahrverhaltens zu erhalten. Ob darüber hinaus die seit geraumer Zeit erhobene Forderung der Ausgestaltung des Fahrverbots als deliktsunabhängige Sanktion sinnvoll erscheint, ist dagegen äußerst fragwürdig. Gerade der Zusammenhang mit dem Führen eines Kraftfahrzeugs i.S.v. $\int 44$ StGB führt dem jungen Täter sein fehlerhaftes Verkehrsverhalten deutlicher vor Augen als bei einem deliktsunabhängigen Fahrverbot. So bekommt er die Konsequenzen direkt zu spüren, nämlich dass er in seiner Mobilität eingeschränkt ist. Weiterhin muss beachtet werden, dass das Fahrverbot überwiegend Kriminalität im unteren oder mittleren Bereich betrifft, so dass hiervon vorwiegend berufstätige oder sich in der Ausbildung befindliche Heranwachsende betroffen sind. Jedenfalls sollte die Jugendtypik gewisser Verhaltensweisen gerade hinsichtlich der Straßenverkehrsdelikte bei der Anordnung einer Sanktion stets Beachtung finden, da sie oft aus einer situationsspezifisch unüberlegten Kurzschlussreaktion resultieren sowie Folge einer noch nicht abgeschlossenen Persönlichkeitsbildung sind.

Die Autorin ist wissenschaftliche Hilfskraft in Jena (bei Prof. Dr. Frank Neubacher)

\section{Fußnoten:}

1 Böcher, NZV 89, 1 (2).

2 Walkenhorst, ZJJ 03, 164 (168).

3 Mettke, Jura 00, 145 (154).

4 2. Periodischer Sicherheitsbericht, S.319.

5 Böcher, NZV 89, 1 (3).

6 Böcher, NZV 89, 1 (3).

7 Böcher, NZV 89, 1 (3); Müller, NZV 00, 401 (405); Yorck Herzberg/ Schlag, ZfSozpsych 06, 73 (74).

8 Yorck Herzberg/Schlag, ZfSozpsych 06, 73 (79).

9 BT-DR 16/5047, S. 7.

10 Kreuzer, NJW 02, 2345 (2349/2350).

11 Yorck Herzberg/Schlag, ZfSozpsych 06, 73 (79).

12 Lösel/Bliesener, Aggression u. Delinquenz unter Jugendlichen, S. 9.

13 Walter, JugendKrim., 3.Aufl. 2005, S. 184 Rn. 131.

14 Lösel/Bliesener, Aggression u. Delinquenz unter Jugendlichen, S. 152.

15 Jehle/Hohmann-Fricke, ZJJ 06, 286 (289).

16 Reicher, Kindheit und Entwicklung 99, 171 (173).

17 Eisenberg, JGG, $\mathbb{1 0 5}$ Rn. 31.

18 Jehle/Hohmann-Fricke, ZJJ 06, 286 (288).

19 Eisenberg, JGG, $\mathbb{\$} 105$ Rn. 31.

20 Eisenberg, JGG, $\mathbb{1 0 5}$ Rn. 31.

21 NJW 60, 1966 (1966).

22 www.conti-online.com/Pressemitteilungen/Presseservice/26.04.2006.

23 Hufnagel, NJW 07, 2577 (2578).

24 Eisenberg, JGG, $\mathbb{1} 105$ Rn. 2.

25 BGH NStZ 87, 366 (366); Hinz, ZRP 01, 106 (107).

26 BGH NStZ 87, 366 (366).

27 OLG Zweibrücken NZV 89, 442 (442).

28 BGH NStZ-RR 99, 26 (26).

29 AG Saalfeld ZJJ 03, 307 (308).

30 OLG Zweibrücken NZV 89, 442 (442).

31 OLG Hamm NJW 60, 1966 (1967).

32 OLG Hamm NJW 60, 1966 (1967).

33 AG Saalfeld, NStZ 94, 89 (90).

34 AG Saalfeld, NStZ 94, 89 (90); Ostendorf, JugendstrR, 4. Aufl. 2007, Rn. 141,142 .

35 BGH NStZ-RR 99, 26 (26); BGHSt 36, 37(40).

36 Wölfl, JuS 02, 75 (77).

37 BGH NStZ-RR 03, 186 (186); BGH NStZ 04, 294 (294).

38 Sonnen in Diemer/Schoreit/Sonnen, JGG, $\mathbb{\$} 105$ Rn. 15; Eisenberg, JGG, $\mathbb{\$} 105$ Rn. $18,19 \mathrm{~b}$.

39 Ostendorf; JGG, $\mathbb{} 105$ Rn.7; Sonnen in Diemer/Schoreit/Sonnen, JGG, $\$ 105 \mathrm{Rn} .17$.

40 Eisenberg, JGG, $\mathbb{\$} 105$ Rn. 31. 